\title{
Candida albicans EN PACIENTES CON ESTOMATITIS SUBPROTÉSICA DEL CENTRO DEL ADULTO MAYOR DE CHICLAYO, PERÚ
}

\author{
Candida albicans IN PATIENTS WITH DENTURE STOMATITIS OF THE CENTER FOR \\ THE ELDERLY ADULT, CHICLAYO, PERÚ
}

Hugo Antonio Álvarez Chupillón

\author{
RESUMEN
}

Objetivo: Determinar si hay asociación entre la presencia de Candida albicans y el grado de estomatitis subprotésica (ESP) en pacientes del Centro del Adulto Mayor (CAM) de Chiclayo (julio, 2012). Materiales y Métodos: Se evaluaron a 58 pacientes con prótesis dentales removibles diagnosticados clínicamente con ESP. Se les realizó un hisopado de la mucosa palatina para luego ser cultivados directamente en medio de cultivo CHROMagar Candida a $37^{\circ} \mathrm{C}$ por 48 horas. Los cultivos que dieron positivos para Candida albicans fueron sometidos a la prueba del tubo germinal. Resultados: Un total de 35 cultivos (60\%) dieron positivo a Candida albicans, siendo el grado III de ESP el que presentó un $100 \%$ de asociación a Candida albicans $(n=3)$. El grado II presentó $70,8 \%(n=17)$. La prótesis total estuvo más comprometida con presencia de Candida albicans $(65,5 \%)$ sin significancia estadística $(p=0,591)$. Conclusiones: Existe asociación entre los grados de ESP y Candida albicans.

Palabras clave: Candida albicans; estomatitis subprotésica; adulto mayor; prótesis dental. (Fuente: DeCS BIREME)

\begin{abstract}
Objective: Determine if there is an association between the presence of Candida albicans and the denture stomatitis type in patients who attend to the aged center of Chiclayo - July 2012. Materials and Methods: 58 patients with removable dental prostheses diagnosed clinically with denture stomatitis were evaluated. A palatal mucosal swab was performed and then cultured directly in the medium of the CHROMagar Candida culture at $37^{\circ} \mathrm{C}$ for 48 hours. The crop that tested positive for Candida albicans were submitted to germ-tube testing. Results: A total of $35(60 \%)$ cultures tested positive for Candida albicans, with grade III of denture stomatitis being $100 \%$ associated with Candida albicans $(\mathrm{n}=3)$, grade II presented $70.8 \%(n=17)$. The total prosthesis was more involved in the presence of Candida albicans (65.5\%) with no statistical significance, $p=0.591$. Conclusions: There is association between the denture stomatitis and Candida albicans.
\end{abstract}

Key words: Candida albicans; denture stomatitis, aged, dental prosthesis (Source: MeSH NLM)

Recibido: 17 de octubre de 2017

${ }^{1}$ Universidad San Martin de Porres - Filial Norte

a Cirujano dentista.

Correspondencia:

Hugo Antonio Àlvarez Chupillón

Correo electrónico: hugo ant@hotmail.com

\section{๑(1) $\odot($}




\section{INTRODUCCIÓN}

En la actualidad, la mayoría de los adultos mayores portadores de prótesis dentales presentan alteraciones en la mucosa bucal. Esto puede deberse a la acción de cubrir la mucosa masticatoria, lo que produce presión, tracción y puede llevar a irritación tisular. Estos efectos pueden alterar la homeostasis y derivar en una estomatitis subprotésica ${ }^{(1)}(E S P)$.

La ESP se caracteriza por presentar la mucosa inflamada, brillante, eritematosa, y eventualmente hemorrágica, limitada a los tejidos en contacto directo con la dentadura(2). La ESP se clasifica en tres grados: Grado I (inflamación simple localizada), Grado II (inflamación simple generalizada) y Grado III (inflamación granular o papilar hiperplásica) ${ }^{(3,4)}$.

Se calcula que el $95 \%$ de los adultos mayores portadores de prótesis dentales por más de un año presentan cambios citológicos en la mucosa de soporte ${ }^{(5)}$.

El principal factor etiológico de ESP es la presencia de Candida albicans que es un hongo polimórfico comensal inofensivo, que bajo ciertos factores pueden transformarse en patógenos oportunistas y causar infecciones ${ }^{(6)}$.

Un factor predisponente es la acción de cubrir la mucosa masticatoria con una prótesis dental, la cual dificulta la llegada de anticuerpos salivales, condicionan la creación de un medio ácido y anaerobio que favorece la proliferación, adhesión y patogenicidad, activando fosfolipasas extracelulares y proteinasas ácidas de Candida que generan metabolitos que alteran algunos mecanismos inmunológicos.

Otros factores como la rugosidad de la superficie, película salival, la energía libre de la superficie y el tipo de material de la prótesis, contribuyen aún más en su virulencia $(7,8,9)$.

El objetivo de esta investigación fue determinar la asociación entre la presencia de Candida albicans y el grado de estomatitis subprotésica (ESP).

\section{MATERIALES Y MÉTODOS}

Estudio observacional, descriptivo, transversal y prospectivo. Se realizó en el Centro del Adulto Mayor (CAM) de Chiclayo (Lambayeque), que cuenta con una población aproximada de 100 usuarios. La muestra estuvo conformada por 58 adultos mayores de ambos sexos portadores de prótesis dentales, que asistieron al CAM, en el periodo de julio del 2012.

La muestra fue intencional y se consideraron los criterios de inclusión: adultos mayores portadores de prótesis dentales superiores que presentaban algún signo de ESP en la mucosa palatina; adultos mayores que se encontraban en buen estado general sin tratamiento médico antifúngico que aceptaran participar en el estudio y firmaran el consentimiento informado.

Con la autorización de la directora del CAM de Chiclayo, se realizó el examen de la cavidad bucal a los sujetos seleccionados mediante la utilización de un espejo bucal plano, gasa, hisopos estériles. Se empleo materiales de bioseguridad como guantes y mascarilla. El examen se realizó con luz natural y artificial.

Para la recolección de información, se les confeccionó una ficha clínica registrando la edad, género, frecuencia del uso de sus prótesis, con el fin de que la metodología a seguir pueda llegar a determinar factores que predispongan a la etiología de la enfermedad.

El medio de cultivo CHROMagar ${ }^{\mathrm{TM}}$ Candida (medio selectivo cromogénico con una especificidad y sensibilidad para Candida albicans del 99\%), según las indicaciones del fabricante $(47,7 \mathrm{~g} / \mathrm{L}$ para $1000 \mathrm{ml}$. de agua purificada), fue elaborado en el Laboratorio de Microbiología de la Universidad San Martin de Porres - Filial norte (USMP - FN).

Se realizó un hisopado de la mucosa palatina donde se observó clínicamente los signos de ESP en cualquiera de sus grados y se sembró directamente en el medio CHROMagar ${ }^{\mathrm{TM}}$ Candida, para luego llevar las muestras al Laboratorio de Microbiología de la USMP-FN para incubarlas a $37^{\circ} \mathrm{C}$, por 48 horas en condiciones de aerobiosis.

Al cabo de 48 horas, se evidenció el crecimiento de colonias de un color verde, redondeadas, ovoidales, elevadas y de bordes enteros, que según el fabricante especificaban la presencia de Candida albicans. (Figura 1).

Prueba de confirmación: Todas las colonias de color verde fueron sometidas a la prueba de tubo germinativo, la cual consistió en tomar un inóculo de la colonia de Candida albicans con el asa de Khole, el cual se inoculó en $0,5 \mathrm{ml}$ de suero humano contenido en un tubo de ensayo (Figuras 2 y 3 ). 

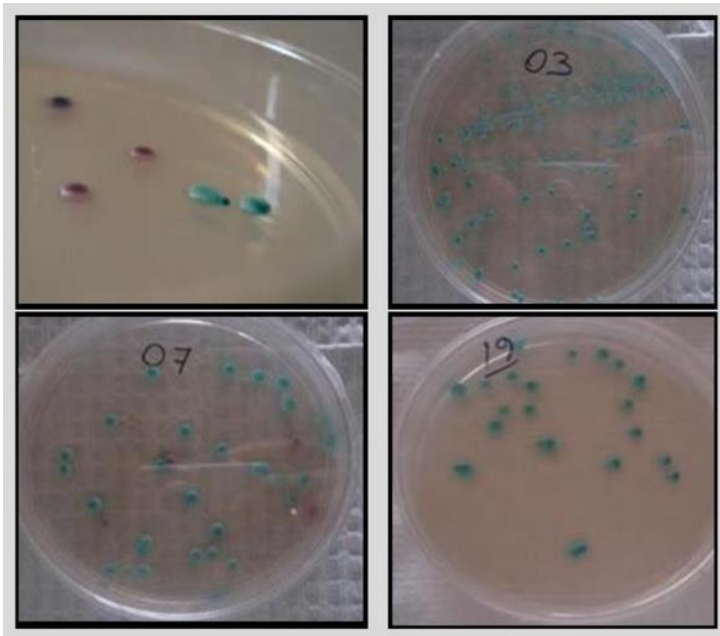

Figura 1. Crecimiento de colonias de la especie Candida albicans en el medio CHROMagar Candida.
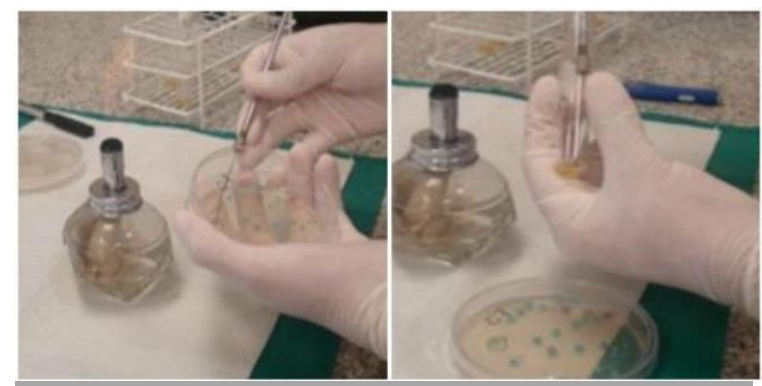

Figura 2. A. Prueba de tubo germinativo. B. Obtención del inoculo de la colonia de C. Albicans.

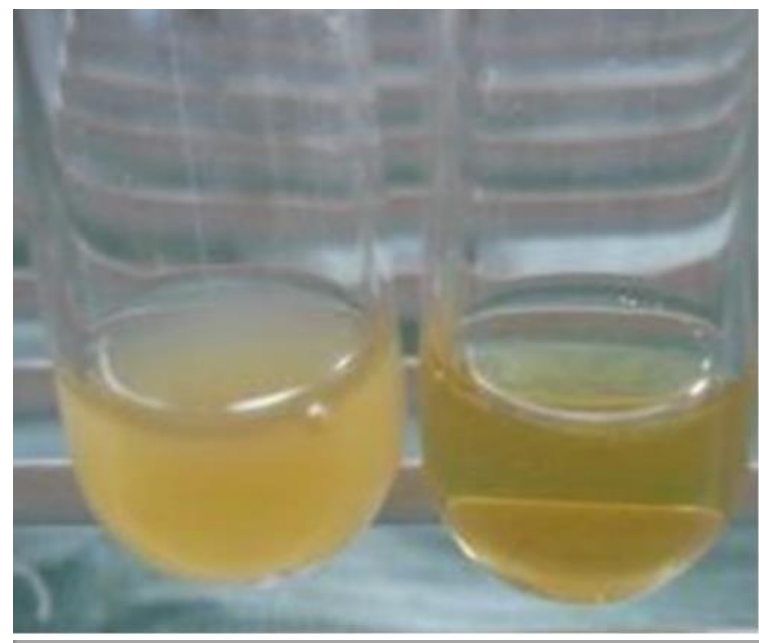

Figura 3. Comparación de suero con inoculo de cándida (izquierda) y suero humano (derecha).
Se incubó a $37^{\circ} \mathrm{C}$ por 3 horas. Al término del tiempo, se realizó una observación en fresco con microscopio óptico, empleando los lentes de aumento de 10x y 40x para observar la formación de tubos germinales (Figura 4) y así confirmar la presencia de Candida albicans.

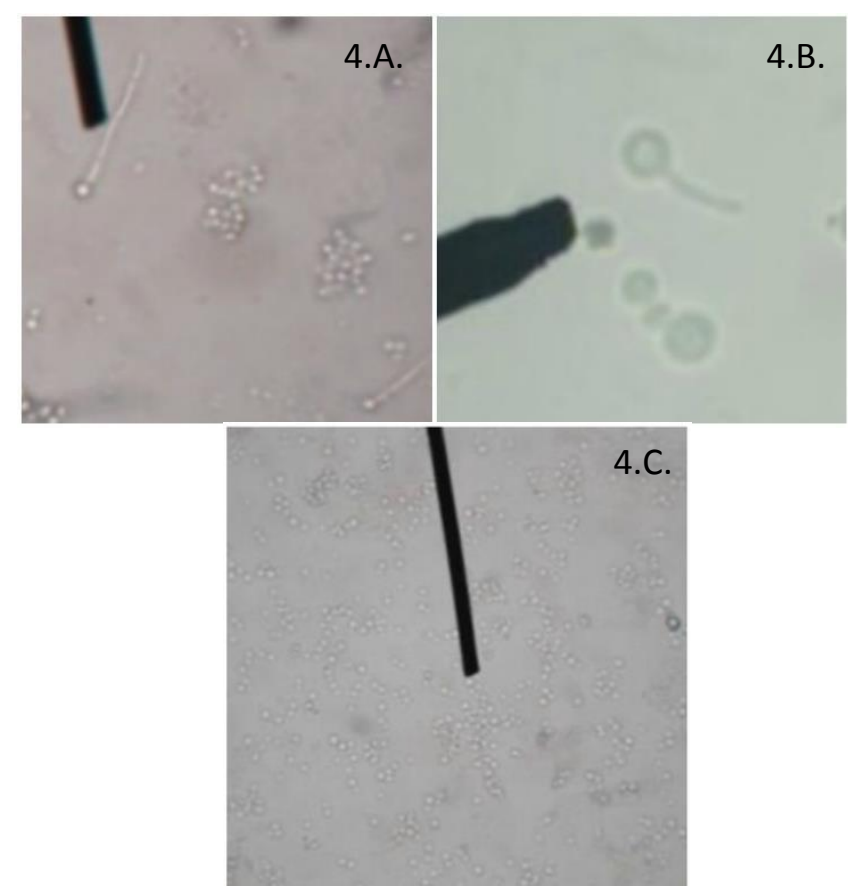

Figura 4. A. Formación de tubo germinal (aumento de 10x.) Figura B y C: Formación de tubo germinal (aumento de 40x).

Al concluir con la presente investigación, se realizó una charla informativa para todos los pacientes de la muestra del estudio, explicándoles brevemente en qué consiste la ESP, con el fin de concientizarlos a mejorar su salud bucal y a tomar medidas preventivas.

Los datos fueron analizados en un paquete estadístico SPSS V. 20.0. Se realizaron las pruebas estadísticas de Chi cuadrado con corrección de Yates y Tau de Goodman y Kruska.

\section{RESULTADOS}

Este estudio encontró que a mayor severidad del grado de ESP, hay una mayor asociación a Candida albicans $(p=0,047)$. (Tabla 1). 
Tabla 1. Relación entre el grado de estomatitis subprotésica y Candida albicans en pacientes del Centro del Adulto Mayor del distrito de Chiclayo.

\begin{tabular}{|c|c|c|c|c|c|c|}
\hline & & & & & & \\
\hline & & & \multicolumn{3}{|c|}{ Grado DE ESP } & \multirow{2}{*}{ Total } \\
\hline & & & Grado I & Grado II & Grado III & \\
\hline \multirow{6}{*}{$\begin{array}{l}\text { PRESENCIA DE } \\
\text { CANDIDA } \\
\text { ALBICANS }\end{array}$} & \multirow[t]{2}{*}{ No } & Pacientes & 16 & 7 & 0 & 23 \\
\hline & & $\%$ & 51,6 & 29,1 & 0 & 39,7 \\
\hline & \multirow{3}{*}{ Si } & Pacientes & 15 & 17 & 3 & 35 \\
\hline & & $\%$ & 48,4 & 70,8 & 100 & 60,3 \\
\hline & & Pacientes & 31 & 24 & 3 & 58 \\
\hline & Total & $\%$ & 100 & 100 & 100 & 100 \\
\hline
\end{tabular}

Fuente: Centro del Adulto Mayor del distrito de Chiclayo.

Según el tipo de prótesis dentales, el $65,5 \%$ de las prótesis totales están asociadas a Candida albicans. La prueba no es significativa $(p=0,591)$ (Tabla 2$)$.

Tabla 2. Relación entre el material de la prótesis dental y candida albicans en pacientes del Centro del Adulto Mayor del distrito de Chiclayo.

\begin{tabular}{|c|c|c|c|c|c|}
\hline & & & \multirow{2}{*}{\multicolumn{2}{|c|}{ MATERIAL PRÓTESIS DENTAL }} & \multirow{3}{*}{ - Tota } \\
\hline & & & & & \\
\hline & & & Acrílica & Metálica & \\
\hline \multirow{6}{*}{$\begin{array}{l}\text { PRESENCIA DE } \\
\text { CANDIDA } \\
\text { ALBICANS }\end{array}$} & \multirow{2}{*}{ No } & Pacientes & 18 & 5 & 23 \\
\hline & & $\%$ & 40,9 & 35,7 & 39,7 \\
\hline & \multirow{2}{*}{ Sí } & Pacientes & 26 & 9 & 35 \\
\hline & & $\%$ & 59,1 & 64,3 & 60,3 \\
\hline & \multirow{2}{*}{ Total } & Pacientes & 44 & 14 & 58 \\
\hline & & $\%$ & 100 & 100 & 100 \\
\hline
\end{tabular}

Fuente: Centro del Adulto Mayor del distrito de Chiclayo.

Se observa que del total, el material acrílico está asociado a Candida albicans en un $59,1 \%$ y el material metálico, en un $64,3 \%$. La prueba no es significativa $(p=0,974)$.

\section{DISCUSIÓN}

La ESP es una enfermedad que va en aumento. Dentro de la literatura revisada sobre la prevalencia de la misma, encontramos que Nápoles et al. ${ }^{(10)}$ y Ley et al. ${ }^{(11)}$ concluyeron que el $100 \%$ de su muestra de estudio presentó ESP. Por otro lado, la ESP se agrava más con la presencia de Candida albicans, como bien lo manifiestan el estudio mexicano de Hernández ${ }^{(12)}$, el estudio peruano de $\operatorname{Rojas}^{(3)}$, el estudio de Pan et al..$^{(9)}$, la investigación brasileña de Gauch et al. ${ }^{(13)}$ y las investigaciones chilenas de Mardones ${ }^{(14)}$ y de Brevis et al. ${ }^{(8)}$ quienes coincidieron en concluir que existe una fuerte asociación entre Candida y la ESP, siendo la Candida albicans la levadura más frecuentemente aislada de los adultos mayores con ESP y asociada a su patogenicidad.

Sin embargo, el estudio de Carmona et al. concluyò que el género Cándida se aisló en un porcentaje bajo, planteando que estos microorganismos no siempre son los agentes etiológicos de la estomatitis subprotésica ${ }^{(15)}$.

Este trabajo de investigación se realizó sólo en adultos mayores que ya presentaban algún signo de ESP en cualquiera de sus grados. Como bien refiere la literatura, mientras más avance el grado de ESP, la Candida albicans agrava el cuadro clínico $(16,17)$. Sobre esta base, este estudio determinó que la ESP grado II fue la más frecuente, siendo el grado III el que presentó un $100 \%$ de asociación a Candida albicans (Tabla 1).

La prótesis total representó un $65,5 \%$ de asociación a Candida albicans (Tabla 2). Esta predominancia concuerda con lo estudiado por Lazarde et al. ${ }^{(16)}$ y Rojas ${ }^{(3)}$. Esto se debe, según el estudio de Pineda et al. a que la Candida albicans a partir de las 11 horas de inoculación empieza a aglutinarse no solo en las células del hospedero sino en materiales inertes como en las áreas irregulares de la resina acrílica, lo cual crea reservorios protegidos para el hongo( ${ }^{(18)}$.

Los resultados mostraron la existencia de asociación entre Candida albicans y el grado de estomatitis subprotésica, lo cual corrobora los antecedentes hallados para la presente investigación.

Se concluye que existe asociación entre la presencia de Candida albicans y el grado de Estomatitis subprotésica. No se encontró asociación entre el tipo de prótesis y la presencia de Candida albicans.

Se recomienda realizar estudios con una muestra mayor y con un diseño longitudinal y aleatorio simple; así mismo, realizar actividades educativas relacionadas con las complicaciones por no cumplir con el adecuado mantenimiento de las prótesis dentales.

\section{Agradecimientos:}

A la Lic. Obs. Mariela Ladera Nieto.

Contribuciones de autoría: $\mathrm{HACH}$ participó en el diseño del estudio, recolección y análisis de datos. Redactó y aprobó el artículo.

\section{Fuente de financiamiento:}

Financiado por el autor.

\section{Conflictos de interés:}

El autor declara no tener conflicto de interés en esta publicación.

\section{REFERENCIAS BIBLIOGRÁFICAS}

1. Díaz Martell Yensi, Martell Forte lleana de la Caridad, Zamora Díaz Javier Domingo. Afecciones de la 
mucosa oral encontradas en pacientes geriátricos portadores de prótesis estomatológicas. Rev Cubana Estomatol [Internet]. 2007 Sep [citado 17 de stiembre de 2017 17] ; 44( 3 ): . Disponible en: http://scielo.sld.cu/scielo.php?script=sci_arttext\&pid= S0034-75072007000300002\&Ing=es.

2. Pardi G, Cardozo E. Relación entre placa dental y estomatitis subprotésica. Acta Odontológica (Venezuela) [Internet] 2003. [citado 14 de setiembre de 2017]; 41(1). Disponible en: http://www.actaodontologica.com/ediciones/2003/1/pl aca_dental_estomatitis_sub_protesica.asp

3. Rojas L. Especies del género Cándida implicadas en estomatitis subprotésica de pacientes del Departamento de Odontoestomatología del Centro Médico Naval "CMST"-2007. [Tesis de licenciatura]. Lima: Universidad Nacional Mayor De San Marcos; 2008.

4. Noguera, G; Fleitas, A. Frecuencia de la estomatitis subprotésica en pacientes portadores de dentaduras totales. Revista Odontológica de Los Andes (Venezuela) [Internet] 2005. [citado 13 de setiembre de 2017]; 1(1). Disponible en: http://erevistas.saber.ula.ve/index.php/odontoula/articl e/view/7295/7166

5. Vilchez Salazar E, Watanabe Velásquez R, Reyes Soto S, Vilchez Quintana E, Torres Roque C, Vilchez Quintana M. Lesiones subprotésicas en pacientes portadores de prótesis completa. Odontol Sanmarquina [Internet] 2013. [citado 13 de setiembre de 2017]; 16(1). Disponible en: http://revistasinvestigacion.unmsm.edu.pe/index.p hp/odont/article/view/5371/4605...5

6. Mayer FL, Wilson D, Hube B. Candida albicans pathogenicity mechanisms. Virulence [Internet] 2013. [citado 15 de setiembre de 2017] 4(2):119-28. Disponible en: https://www.ncbi.nlm. nih.gov/pmc/articles/PMC3654610/

7. Salerno C, Pascale M, Contaldo M, Esposito V, Busciolano M, Milillo L, Guida A, Petruzzi M, Serpico R. Candida-associated denture stomatitis. Med Oral Patol Oral Cir Bucal [Internet] 2011 Mar 1. [citado 17 de setiembre de 2017]; 16(1): 139-43. Disponible en: http://www.medicinaoral.com/medoralfree01/v16i2/me doralv16i2p139.pdf

8. Brevis P, Cancino J, Cantín M. Estomatitis subprótesis: Estudio clínico y microbiológico de Cándida. Int. J. Odontostomat 2008; 2(1): 101-08

9. Pan H, Wang G, Pan J, Ye G, Sun K, Zhang J, Wang J. Cold plasma-induced surface modification of heatpolymerized acrylic resin and prevention of early adherence of Candida albicans. Dental Materials Journal (China) [Internet] 2015. [citado 18 de setiembre de 2017]; 34(4): 529-36. Disponible en: https://www.jstage.jst.go.jp/article/dmj/34/4/34_2015035/_pdf
10. Nápoles I, Barciela J, Cabrera N, Puig E. Eficacia del tratamiento homeopático en la estomatitis subprótesis. Archivo Médico de Camagüey. Centro Provincial de Ciencias Médicas de Camagüey [Internet] 2008. [citado 18 de setiembre de 2017]; 12(5). Disponible en: http://scielo.sld.cu/pdf/amc/v12n5/amc07508.pdf

11. Ley L, Silva Y, Martín O, Paz E, Landrián C. Eficacia del aceite de girasol ozonizado en el tratamiento de la estomatitis subprótesis grado I y II. Archivo Médico de Camagüey. Centro Provincial de Ciencias Médicas de Camagüey [Internet] 2008. [citado 09 de setiembre de 2017] ;12(3):1-9. Disponible en: http://scielo.sld.cu/pdf/amc/v12n3/amc05308.pdf

12. Hernández S, Villamil J, Lama E, Puc R, Rueda F. Prevalencia de Cándida albicans en pacientes con y sin estomatitis subprotésica. Rev odontol latinoam (México). 2009; 1(1): 7-11.

13. Gauch LM, Pedrosa SS, Silveira-Gomes F, Esteves RA, Marques-da-Silva SH. Isolation of Candida spp. from denture-related stomatitis in Pará, Brazil. Braz J Microbiol [Internet] 2017. [citado 05 de setiembre de 2017]. Disponible en: http://dx.doi.org/10.1016/j.bjm.2017.07.001

14. Mardones P. Búsqueda e identificación de candida albicans en pacientes con estomatitis subprotésica atendidos en el centro de clínicas odontológicas de la Universidad de Talca. Talca: Universidad de Talca; 2005.

15. Carmona M, Pulido M, Abud K, Bolivar K, Cortés $\mathrm{Y}$. Presencia de Candida en pacientes con estomatitis subprotésica que acuden a la Facultad de Odontología de la Universidad de Cartagena en el periodo transcurrido entre agosto 2006 - junio 2007. Revista de la Facultad de Ciencias de la Salud. Universidad de Magdalena (Colombia). 2008; 5(2): 90 - 98.

16. Lazarde J. Estomatitis Subprotésica. Acta Odontol Venez [Internet] 2001. [citado 04 de setiembre de 2017]; 39(3): 9-17. Disponible en: http://www.scielo.org.ve/scielo.php?script=sci_arttext \&pid=S0001-63652001000300003\&Ing=es.

17. Lee Munoz X, Cajas N, Gómez Carranza L, Vergara Núñez C, Ivankovic Silva M, Astorga Bustamante E. Ocurrencia de levaduras del género Candida y estomatitis protésica antes y después del tratamiento rehabilitador basado en prótesis removible. Rev Clin Periodoncia Implantol Rehabil Oral [Internet] 2015. [citado 07 de setiembre de 2017]; 8(1): 31-37. Disponible en

18. Pineda SE, Mosquera J. Adherencia de Candida albicans a resinas acrílicas y poliamidas. Estudio in vitro. Revista Biosalud (Colombia) [Internet] 2017. [citado 05 de setiembre de 2017]; 16(1): 43-50. Disponible

en: http://www.scielo.org.co/pdf/biosa/v16n1/v16n1a06.pd 